DR SAAD A.K.S. AMER (Orcid ID : 0000-0001-8321-1302)

Received Date : 01-Jan-2017

Revised Date : 05-Mar-2017

Accepted Date : 11-Mar-2017

Article type : Systematic review

\title{
Ovarian reserve after salpingectomy: a systematic review and meta-analysis
}

Running headline: Ovarian reserve and salpingectomy

\begin{abstract}
Ahmed Aboelfadle MOHAMED ${ }^{1,3}$, Ali Haroun YOSEF $^{2,3}$, Cathryn JAMES ${ }^{4}$, Tarek Khalaf AL-HUSSAINI $^{3}$, Mohamed Ali BEDAIWY ${ }^{2} \&$ Saad Ali AMER ${ }^{1}$
\end{abstract}

${ }^{1}$ Department of Obstetrics and Gynecology, University of Nottingham, Royal Derby Hospital, Derby, UK

${ }^{2}$ Department of Obstetrics and Gynecology, The University of British Columbia, Vancouver, BC, Canada

${ }^{3}$ Department of Obstetrics and Gynecology, Asyut University, Asyut, Egypt

${ }^{4}$ Derby Teaching Hospitals NHS Foundation Trust, Derby, UK

\section{Corresponding author:}

Saad A K S Amer

School of Medicine, University of Nottingham, Royal Derby Hospital Centre, Uttoxeter Road, Derby DE22 3DT, UK

Email: saad.amer@nottingham.ac.uk

This article has been accepted for publication and undergone full peer review but has not been through the copyediting, typesetting, pagination and proofreading process, which may lead to differences between this version and the Version of Record. Please cite this article as doi: 10.1111/aogs. 13133

This article is protected by copyright. All rights reserved. 
Conflict of interest notification: The authors have nothing to disclose

\begin{abstract}
Introduction: Although there has been a growing concern over the possible damaging effect of salpingectomy on ovarian reserve, this issue remains uncertain. The purpose of this metaanalysis was to test the hypothesis that salpingectomy may compromise ovarian reserve. Material and methods: A detailed search was conducted using MEDLINE, Embase, Dynamed Plus, ScienceDirect, TRIP database and the Cochrane Library from January 2000 to November 2016. All cohort, cross-sectional and randomized controlled studies investigating changes in circulating anti-Müllerian hormone (AMH) after salpingectomy were considered. Thirty-seven studies were identified, of which eight were eligible. Data were extracted and entered into RevMan software for calculation of the weighted mean difference (WMD) and 95\% confidence interval (CI). Two groups of studies were analyzed separately including group 1 (six studies, $\mathrm{n}=464$ ) comparing data before and after salpingectomy and group 2 (two studies) comparing data in women who have undergone salpingectomy $(\mathrm{n}=169) v s$. healthy controls $(\mathrm{n}=154)$. Results: Pooled results of group 1 studies showed no statistically significant change in serum AMH concentration after salpingectomy (WMD, $-0.10 \mathrm{ng} / \mathrm{ml} ; 95 \% \mathrm{CI} ;-0.19-0.00, I^{2}=0 \%$ ). Similarly, meta-analysis of group 2 showed no statistically significant difference in serum AMH concentration between salpingectomy group and controls (WMD, $-0.11 \mathrm{ng} / \mathrm{ml}$; $95 \% \mathrm{CI}$; $0.37-0.14, I^{2}=77 \%$ ). Subgroup analyses based on laterality of surgery, type of AMH kit and participants' age $(<40)$ still showed no statistically significant changes in circulating AMH. Conclusion: Salpingectomy does not seem to compromise ovarian reserve in the short-term. However, the long-term effect of salpingectomy on ovarian reserve remains uncertain.
\end{abstract}

\title{
Keywords
}

salpingectomy, ovarian reserve, anti-Müllerian hormone, ovarian function, laparoscopy. 


\author{
Abbreviations \\ AMH anti-Müllerian hormone \\ FSH follicle stimulating hormone \\ AFC antral follicle count \\ WMD weighted mean difference \\ CI confidence interval \\ IVF in vitro fertilization \\ RCT randomized controlled trials
}

\title{
Key Message
}

This meta-analysis investigates the possible damaging effect of salpingectomy on ovarian reserve. The results confirmed the short-term safety of salpingectomy with no effect on circulating anti-Müllerian hormone, which is the main marker of ovarian reserve

\section{Introduction}

Since its introduction by Lawson Tait in 1883, salpingectomy has been one of the most commonly performed surgical procedures in gynecological practice worldwide (1). Common indications include ectopic pregnancy and salpingitis (for example symptomatic hydro- or pyosalpinx). More recently, it has been widely accepted that salpingectomy should be offered to women with asymptomatic hydrosalpinx who require in vitro fertilisation (IVF) (2). Moreover, with emerging evidence suggesting a tubal origin for ovarian cancer, salpingectomy is increasingly performed during hysterectomy in women conserving their ovaries $(3,4)$.

This rising trend in salpingectomy has been associated with a rising concern over its potential damaging effect on ovarian reserve due to possible concomitant damage of ovarian blood supply given the close proximity between tubal and ovarian arteries. It has therefore been hypothesized that salpingectomy could interrupt ovarian blood supply thereby compromising ovarian blood flow with a consequent decline in ovarian reserve. This hypothesis is supported 
by a previous study showing post-salpingectomy impairment of the ovarian blood flow on the operated side (5). Another study detected an increase in vascular resistance in ovarian arteries following Filshie clip sterilization (6).

As most women requiring salpingectomy are relatively young and still wishing to preserve their fertility potential, it will be critical to evaluate any possible impairment of their ovarian reserve. This will help both the clinician and the patient when considering the need for salpingectomy.

Although there are numerous markers for ovarian reserve, it is now universally agreed that circulating anti Müllerian hormone (AMH) is considered the most reliable test. It has been found to correlate well with the actual histological count of the ovarian follicles (7).

Furthermore, the stability of serum AMH level throughout the menstrual cycle with minimal variations makes it an ideal marker for detecting relatively small changes in ovarian reserve following salpingectomy (8).

To date, there are several studies evaluating the effect of salpingectomy on ovarian reserve (922). Given the small size of these studies, further evidence is required to address this important topic. This meta-analysis was therefore designed to investigate the impact of salpingectomy on ovarian reserve as determined by serum $\mathrm{AMH}$ concentrations.

\section{Material and methods}

This analysis was conducted according to the Preferred Reporting Items for Systematic Reviews and Meta-analyses (PRISMA) guidelines (23) and was registered in PROSPERO (Registration number: CRD42016052576).

\section{Eligible criteria}

We considered all published cohort (retrospective and prospective) and cross-sectional studies as well as randomized controlled trials (RCTs) that investigated changes in serum AMH levels following salpingectomy. 


\section{Outcome measures}

Primary measure

This included changes in the serum AMH concentration after salpingectomy.

Secondary measures

This included changes in serum follicle stimulating hormone (FSH) concentration and antral follicle count (AFC) after salpingectomy.

\section{Search strategy}

A detailed electronic search was conducted using numerous databases from January 2000 to November 2016 to identify available research articles investigating the effect of salpingectomy on ovarian reserve as estimated by changes in serum AMH levels (Supporting Information Table S2). Searched databases included MEDLINE, Embase, Dynamed Plus, ScienceDirect, TRIP database and the Cochrane Library. Medical Subject Headings (MeSH) terms used included: Salpingectomy, Ovarian Function, Ovarian Reserve, Pregnancy, Laparoscopy, Laparotomy, Anti-Müllerian Hormone, Follicle Stimulating Hormone and Fertility. Keywords searched included: Antral Follicle Count, Bilateral Salpingectomy, and Unilateral Salpingectomy. Papers were limited to the English Language, Human Studies and Adult Females. All searches were conducted by the first author (AM) and then independently repeated using the same criteria by an accredited clinical librarian (CJ). All related reports were retrieved, and their reference lists were revised manually to categorize further studies. We also considered published abstracts from conferences.

\section{Study selection}

Two investigators (AM, AY) independently screened the title and abstract of all identified articles to assess relevance to our meta-analysis. In case of disagreement, the full text was retrieved and reviewed independently by another author (SA) for a final decision. 


\section{Data extraction}

All retrieved articles were assessed according to a standardized format including; study design, methods, participant characteristics, intervention, and results. Two investigators (AM and AY) scored the studies and collected the information independently. In the case of discrepancies in scoring between the two investigators, a consensus was reached after discussion or after involvement of the senior investigator (SA). In five studies, the mean \pm SD AMH serum concentration was missing $(11,19,20,21,22)$. Two authors provided the missing data (mean $\pm \mathrm{SD}$ $\mathrm{AMH}$, type of $\mathrm{AMH}$ kit and mean $\pm \mathrm{SD}$ age of participants) when contacted via email $(11,21)$ The author of another study was not contactable (20). Authors of the remaining two studies were contacted by email, but no response was received $(19,22)$.

\section{Quality of included studies and risk of bias assessment}

Modified Newcastle-Ottawa scale was utilised for assessing the quality and risk of bias of the included studies. Each article was scored according to three categories including selection (maximum three stars), comparability (four stars), and outcomes (two stars) $(24,25)$. Selection was rated according to recruitment bias, selection of consecutive women and power calculation. Comparability was assessed based on adjustment of analysis for four confounders including women's age $(<40)$, baseline serum AMH $(\geq 3.1 \mathrm{ng} / \mathrm{ml})$, laterality of surgery and associated hysterectomy. Outcome was scored according to completeness of at least threemonth follow-up after surgery. In the current analysis, we have given more weight to comparability factors and used the cut-off level of six stars with a minimum of three stars in the comparability category $(24,25)$. Table 1 shows the results of quality scores of the studies included in this analysis.

\section{Data analysis}

This meta-analysis included two groups of studies. Group 1 involved studies comparing changes of outcomes (AMH, FSH, AFC) before and after salpingectomy. Group 2 included studies comparing outcomes between women who had undergone salpingectomy $v s$. healthy controls. In both groups, mean \pm SD serum AMH and FSH concentrations and AFC were extracted from the individual studies and pooled using RevMan software (Review Manager, 
version 5.1, The Cochrane Collaboration, 2011; The Nordic Cochrane Centre, Copenhagen, Denmark). The weighted mean difference (WMD) was calculated in both groups. Statistical heterogeneity was assessed by chi-squared $\left(\chi^{2}\right)$ test and $I^{2}$ statistics. A $\chi^{2}$ statistic larger than its degree of freedom or an $I^{2}$ higher than 50\% was indicative of significant heterogeneity between studies. When heterogeneity was significant, a random-effect model was used for metaanalysis. Fixed effect meta-analysis was used when there was no significant heterogeneity.

The initial analyses included data from all studies in both groups. Further subgroup analyses of AMH levels were then performed based on the laterality of the excised fallopian tube, AMH kits and age of participants.

\section{Results}

Thirty-seven studies identified through the electronic search were screened for relevance, of which eight were deemed eligible for the meta-analysis (Fig. 1).

\section{Excluded studies}

Of all identified studies, seven did not measure any ovarian reserve marker and were excluded. Nineteen other studies did not use circulating AMH as an ovarian reserve marker and were excluded. Three further studies were excluded due to missing the mean \pm SD AMH data (Supporting Information Table S1). Authors of these studies were contacted to provide the missing data as explained above.

\section{Included studies}

Details of the included eight studies are shown in Table 2 and Table 3.

\section{Study design}

This systematic review included three prospective cohorts $(11,14,21)$, two retrospective cohorts $(10,13)$, one cross-sectional study $(15)$ and two RCTs $(9,12)$. In one RCT, the two arms including women undergoing salpingectomy (with and without removal of mesosalpinx) were combined and used as a cohort study for this meta-analysis (12). In the other RCT comparing 
laparoscopic hysterectomy with and without bilateral salpingectomy, the salpingectomy arm was used as a cohort study and included in our meta-analysis (9).

\section{Participants}

In four studies, participants had normal fallopian tubes, which were removed during a gynecological procedure including hysterectomy $(9,10,21)$, myomectomy or sterilization (12). In three other studies, participants underwent salpingectomy due to tubal ectopic pregnancy $(11,14,15)$. In the remaining study, women underwent salpingectomy due to tubal pathology including Tubo-ovarian abscess, ectopic pregnancy or hydro-salpinx (13).

All studies stated inclusion and exclusion criteria, which were appropriate, except one study which was a conference abstract (11). We successfully obtained missing data after contacting the author of this study.

\section{Salpingectomy surgery}

All participants underwent salpingectomy through laparoscopy except in two studies, which included women undergoing salpingectomy through laparotomy or laparoscopy $(15,21)$. All studies specified the laterality of salpingectomy, which was bilateral in four studies $(9,10,12,21)$ and unilateral in three studies $(11,14,15)$. The remaining study compared unilateral vs. bilateral salpingectomy (13). Both unilateral and bilateral arms were combined and used in the initial analysis. Each arm was then used separately in the sub-analysis (13). Findley and co-workers tended to preserve mesosalpinx during salpingectomy (9). Venturella and co-workers compared salpingectomy with preservation of mesosalpinx (standard salpingectomy) vs. salpingectomy with removal of mesosalpinx (wide technique salpingectomy) (12). Six studies did not report whether or not the mesosalpinx was preserved $(10,11,13,14,15,21)$.

\section{Length of follow up after salpingectomy}

The length of follow up after salpingectomy was three months in four studies $(9,10,12,14)$, four months in one study (11), six months in one study (21), 18 months in one study (15) and not 
specified in one study (13). One study reported two postoperative measurements at four to six weeks and at three months (9).

\section{AMH kits}

Four kits were used to measure serum AMH levels. The modified AMH Gen II enzyme linked immunosorbent assay (ELISA) (Beckman Coulter, Chaska, MN, USA) was used by four studies $(10-12,14)$. The intra and inter-assay coefficients of variation for the AMH were both below $10 \%$, with a detection limit of $0.08 \mathrm{ng} / \mathrm{m}$. Two studies used the AMH ELISA Ansh Labs assay (Ansh Labs, UK) $(9,15)$. The intra and Inter-assay coefficients of variation for the AMH were $0.02(2.22 / 95)$ and $7.81(15.62 / 2)$, respectively, with a detection limit of $0.06 \mathrm{ng} / \mathrm{ml}$. One study measured AMH by an enzyme linked immunosorbent assay using a Bio-Rad iMark microplate absorbance reader with reagent kits from Bio-Rad (Bio-Rad Laboratories Inc, Hercules, CA) (13). The inter-assay coefficient of variability is $10 \%$, and the intra-assay coefficient of variability is $15 \%$. The remaining study measured AMH using a commercially available ELISA kit (Cusabio Biotech Co., Ltd., Hubei, People's Republic of China). The detection sensitivity of this assay was $0.14 \mathrm{ng} / \mathrm{ml}$ and the inter-assay coefficient was $14.2 \%$ (21). AMH values reported in $\mathrm{pmol} / \mathrm{l}$ were converted to $\mathrm{ng} / \mathrm{ml}$ using a universally accepted laboratory formula: $1 \mathrm{ng} / \mathrm{ml}=7.14 \mathrm{pmol} / \mathrm{l}(26)$.

\section{Potential source of bias}

In all included studies, recruitment of participants followed a consecutive fashion. The selection method was clearly defined, rendering it easy to assess selection bias.

\section{Overall pooled results for all studies}

Pooled analysis of group 1 studies $(n=464)$ revealed no statistically significant change in serum AMH levels after salpingectomy (WMD, $-0.10 \mathrm{ng} / \mathrm{ml}$; 95\% confidence interval (CI); -0.19$\left.0.00, \mathrm{I}^{2}=0 \%\right)$ with low heterogeneity between studies (Fig. 2) $(9-12,14,21)$. Similarly, in group 2, overall analysis of two studies showed no statistically significant difference between the salpingectomy group $(\mathrm{n}=169)$ and control group $(\mathrm{n}=154)$ (WMD, $-0.11 \mathrm{ng} / \mathrm{ml} ; 95 \% \mathrm{CI}$; - 
$0.37-0.14, I^{2}=77 \%$ ) with high heterogeneity between studies (Fig. 3) $(13,15)$.

\section{Subgroup analysis}

Laterality of salpingectomy

In group 1 studies, bilateral salpingectomy was reported in four studies including 383 women $(9,10,12,21)$. Pooled analysis of these studies showed no statistically significant change in postoperative serum AMH level (WMD, $-0.10 \mathrm{ng} / \mathrm{ml} ; 95 \% \mathrm{CI} ;-0.20-0.00 ; \mathrm{I}^{2}=0 \%$ ). Analysis of two studies with unilateral salpingectomy $(n=81)$ revealed no statistically significant change in serum AMH concentration after surgery (WMD, $0.10 \mathrm{ng} / \mathrm{ml} ; 95 \% \mathrm{CI} ;-0.45-0.65 ; \mathrm{I}^{2}=0 \%$ ) $(11,14)$.

In group 2 studies, unilateral salpingectomy was reported in two studies $(13,14)$. Pooled analysis showed no statistically significant difference between salpingectomy group $(\mathrm{n}=128)$ and control group ( $\mathrm{n}=154)$ (WMD, $\left.-0.11 \mathrm{ng} / \mathrm{ml} ; 95 \% \mathrm{CI} ;-0.38-0.16 ; I^{2}=79 \%\right)$.

Studies using different AMH assays

In group 1, analysis of four studies ( $\mathrm{n}=346)$ using modified Gen II AMH kit showed no statistically significant changes in AMH concentration after salpingectomy (WMD, $-0.07 ; 95 \%$ CI; -0.17-0.04; $\left.\mathrm{I}^{2}=0 \%\right)(10-12,14)$.

\section{Participants' age}

In group 1, pooled analysis of three studies including 96 participants aged $<40$ revealed no statistically significant change in serum AMH level (WMD, $-0.05 ; 95 \% \mathrm{CI} ;-0.47-0.57 ; \mathrm{I}^{2}=$ $0 \%)(9,11,14)$. In group 2 studies, all participants were aged under 40 and a sub-analysis was therefore not performed $(13,15)$.

Analysis of three studies in group $1(\mathrm{n}=368)$ with participants $>40$ also revealed no statistically significant change in serum AMH concentration (WMD, $-0.10 \mathrm{ng} / \mathrm{ml} 95 \% \mathrm{CI} ;-0.20-0.00 ; I^{2}=$ $0 \%)(10,12,21)$. 


\section{Sensitivity analysis}

Sensitivity analysis in group 1, included the three studies with participants aged $<40(n=96)$, which were also the studies with low risk of bias. The results of this analysis are shown above. In group 2, only one study scored high in the risk of bias assessment and a sensitivity analysis was therefore not performed (15).

\section{Secondary outcomes}

In group 1, three studies including 368 women measured serum FSH concentrations $(10,12,21)$ and two studies ( $\mathrm{n}=265)$ measured AFC after salpingectomy $(10,12)$. Pooled analysis of these studies revealed a statistically significant increase in circulating FSH (WMD, 1.75 IU/L; 95\% CI; $0.69-2.81 ; I^{2}=81 \%$ ), but no significant change in AFC following bilateral salpingectomy (WMD, $-0.30 ; 95 \% \mathrm{CI} ;-0.64-0.05 ; I^{2}=0 \%$ ).

In group 2, two studies measured serum FSH and AFC $(13,15)$. Meta-analysis of these studies showed no significant difference in serum FSH levels between salpingectomy $(n=169)$ and control ( $\mathrm{n}=154)$ groups (WMD, $\left.0.58 \mathrm{IU} / \mathrm{L} ; 95 \% \mathrm{CI} ;-0.21-1.37 ; I^{2}=63 \%\right)$. On the other hand, pooled analysis showed a significantly lower AFC in salpingectomy group compared to the control group (WMD, -0.66; 95\% CI; $-1.27--0.06 ; I^{2}=42 \%$ ).

\section{Discussion}

To the best of our knowledge, this is the first systematic review to investigate the impact of salpingectomy on ovarian reserve as determined by circulating $\mathrm{AMH}$. The overall analysis as well as subgroup analysis based on laterality, age and AMH kits revealed no short-term changes in serum AMH concentrations after salpingectomy.

These results are surprising given the expected post-salpingectomy damage to ovarian reserve as a result of impairment of ovarian blood supply as shown in several previous studies $(5,6,13)$. Furthermore, it was interesting to see that even wide (radical) and bilateral salpingectomy did not cause any decline in serum AMH concentration despite the expected increase in damage to the ovarian blood supply. Our results are in disagreement with a recent systematic review, which reported post-salpingectomy impairment of ovarian reserve as determined by ovarian 
response to hyperstimulation during IVF (27). This discrepancy could be explained by the differences in the methods used to assess ovarian reserve between our meta-analysis (AMH) and the previous systematic review (ovarian response during IVF).

It was also surprising to see that ovarian reserve was not affected after salpingectomy even when performed with hysterectomy despite the loss of an important ovarian blood supply from the utero-ovarian vessels. It may be argued that studies involving hysterectomy should have been excluded from this meta-analysis due to the added element of damage to utero-ovarian vessels. However, we included these studies in order to address a well-recognised concern over the possible adverse effect of opportunistic salpingectomy during hysterectomy on ovarian reserve. We believe this is a clinically important issue that will be of interest to gynecological surgeons. Had we found an effect on AMH in the hysterectomy group we would have performed a sub-analysis to exclude studies involving hysterectomy.

Possible explanation for our unexpected results is that the postulated post-salpingectomy decline of ovarian reserve may be a chronic process that could take a long time to occur. In other words, the possible post-salpingectomy impairment of blood supply may lead to chronic ovarian ischemia that could take a relatively long time to cause reduction in the number of the small antral follicles with subsequent fall in circulating $\mathrm{AMH}$, which is exclusively secreted from these follicles. This hypothesis, however, requires validation through further long-term follow up studies on the changes of circulating AMH after salpingectomy. One recent longterm study revealed no change in circulating AMH up to five years after salpingectomy (28). However, these data are inconclusive due to the relatively old age of all participants (mean age, 44) with very low AMH values at baseline.

As far as other ovarian reserve markers are concerned, group 1 analysis revealed a small but a statistically significant increase in circulating FSH (WMD, 1.75 IU/L). However, given the well-known high inter- and intra-cycle variability of circulating FSH, this little change is unlikely to reflect a real decline in ovarian reserve. Furthermore, serum FSH level is currently considered as an ovarian reserve marker of little clinical value in young women (29). With regards to the AFC, it was interesting to see (in group 2 meta-analysis) lower values in women undergoing salpingectomy compared to the healthy controls. This is surprising as AFC outcome was expected to be in line with $\mathrm{AMH}$ as both correlate well with each other. It is worth mentioning the larger of the two studies in this analysis included participants with tubal/adnexal pathology which could have already compromised AFC before surgery. This is 
supported by the lack of any effect of salpingectomy on AFC in group 1 studies, which included participants with no tubal pathology. However, this does not explain the discrepancy between AFC and AMH outcomes in group 2 meta-analysis. This issue could only be resolved with further studies comparing pre- and post-salpingectomy AFC and circulating AMH in women with and without tubal pathology.

Based on our results, there seems to be no evidence of any short-term compromise to ovarian reserve following salpingectomy. However, these results should be interpreted with caution, given the short duration of follow up in all studies included in this meta-analysis. Furthermore, the lack of any short term decline in serum AMH levels after salpingectomy does not necessarily mean that the long-term chances of pregnancy are not compromised by this procedure. We therefore recommend that until further evidence becomes available from longterm follow-up studies, salpingectomy should only be performed when necessary and women should be counselled with regards to the uncertainty regarding risks to ovarian reserve.

Alternative conservative approaches may be advisable whenever possible.

The main limitations of this meta-analysis are the very small number of the studies included and the short duration of follow-up, which make it difficult to draw any objective conclusion on the long-term preservation of ovarian function after surgery. Another important limitation is the variation in the surgical techniques and tubal pathologies in participants of different studies. Furthermore, although circulating AMH is considered the most reliable marker of ovarian reserve, it does not directly measure the total follicle pool. It should therefore be considered a surrogate marker of ovarian reserve and the results should be interpreted with caution.

With regards to the quality and risk of bias assessment of the included studies, it is worth mentioning that all available tests including the Newcastle-Ottawa scale, which is used in this study, are of limited ability when the number of included studies is small.

In conclusion, salpingectomy seems to have no short-term adverse effect on ovarian reserve. However, given the possible concomitant damage to ovarian blood supply during salpingectomy, long term adverse effect on ovarian reserve remains a concern that requires further investigations. 


\section{Funding}

Funding was obtained from the Egyptian Cultural Centre and Education bureau in London and the British Council in Cairo.

\section{References}

1. Golditch IM. Lawson Tait: the forgotten gynecologist. Obstet Gynecol. 2002;99:152-6.

2. Johnson NP, Mak W, Sowter MC. Laparoscopic salpingectomy for women with hydrosalpinges enhances the success of IVF: a Cochrane review. Hum Reprod. 2002;17:543-8.

3. Tone AA, Salvador S, Finlayson SJ, Tinker AV, Kwon JS, Lee CH et al. The role of the fallopian tube in ovarian cancer. Clin Adv Hematol Oncol. 2012;10(5):296-306.

4. Kwon JS. Ovarian cancer risk reduction through opportunistic salpingectomy. J Gynecol Oncol. 2015;26:83-6.

5. Chan $\mathrm{CC}, \mathrm{Ng} \mathrm{EH}, \mathrm{Li} \mathrm{CF}, \mathrm{Ho}$ PC. Impaired ovarian blood flow and reduced antral follicle count following laparoscopic salpingectomy for ectopic pregnancy. Hum Reprod. 2003;18:2175-80.

6. Sumiala S, Pirhonen J, Tuominen J, Maenpaa J. Increased uterine and ovarian vascular resistance following Filshie clip sterilization: preliminary findings obtained with color Doppler ultrasonography. J Clin Ultrasound. 1995;23:511-6.

7. Hansen KR, Hodnett GM, Knowlton N, Craig LB. Correlation of ovarian reserve tests with histologically determined primordial follicle number. Fertil Steril. 2011;95:170-5.

8. Lambert-Messerlian G, Plante B, Eklund EE, Raker C, Moore RG. Levels of antimüllerian hormone in serum during the normal menstrual cycle. Fertil Steril. 2016;105:208-13.

9. Findley AD, Siedhoff MT, Hobbs KA, Steege JF, Carey ET, McCall CA et al. Short-term effects of salpingectomy during laparoscopic hysterectomy on ovarian reserve: a pilot randomized controlled trial. Fertil Steril. 2013;100:1704-8.

10. Morelli M, Venturella R, Mocciaro R, Di Cello A, Rania E, Lico D et al. Prophylactic salpingectomy in premenopausal low-risk women for ovarian cancer: primum non nocere. Gynecol Oncol. 2013;129:448-51.

11. Rodgers R, Jonathan C, Geoffrey R, Jason A, Michael C, Joanne L. The Impact of Salpingectomy for Ectopic Pregnancy on Ovarian Reserve. J Minim Invasive Gynecol. 2015;22:S75. 
12. Venturella R, Morelli M, Lico D, Di Cello A, Rocca M, Sacchinelli A et al. Wide excision of soft tissues adjacent to the ovary and fallopian tube does not impair the ovarian reserve in women undergoing prophylactic bilateral salpingectomy: results from a randomized, controlled trial. Fertil Steril. 2015;104:1332-9.

13. Ye XP, Yang YZ, Sun XX. A retrospective analysis of the effect of salpingectomy on serum anti Müllerian hormone level and ovarian reserve. Am J Obstet Gynecol. 2015;212:53.e1-10.

14. Sahin C, Taylan E, Akdemir A, Ozgurel B, Taskıran D, Ergenoglu AM. The impact of salpingectomy and single-dose systemic methotrexate treatments on ovarian reserve in ectopic pregnancy. Eur J Obstet Gynecol Reprod Biol. 2016;205:150-2.

15. Sahin Ersoy G, Turhan OT, Sakin O, Bulut S. Comparison of the long-term effects of single-dose methotrexate and salpingectomy on ovarian reserve in terms of anti-müllerian hormone levels. Hum Fertil. 2016;19:262-7

16. Ulug P, Oner G. Evaluation of the effects of single or multiple dose methotrexate administration, salpingectomy on ovarian reserve of rat with the measurement of antiMüllerian hormone (AMH) levels and histological analysis. Eur J Obstet Gynecol Reprod Biol. 2014; 181:205-9.

17. Sezik M, Ozkaya O, Demir F, Sezik HT, Kaya H. Total salpingectomy during abdominal hysterectomy: effects on ovarian reserve and ovarian stromal blood flow. J Obstet Gynaecol Res. 2007;33:863-9.

18. Atilgan R, Kuloglu T, Boztosun A, Orak U, Baspinar M, Can B et al. Investigation of the effects of unilateral total salpingectomy on ovarian proliferating cell nuclear antigen and follicular reserve: experimental study. Eur J Obstet Gynecol Reprod Biol. 2015;188:56-60.

19. Ni L, Sadiq S, Mao Y, Cui Y, Wang W, Liu J. Influence of various tubal surgeries to serum antimullerian hormone level and outcome of the subsequent IVF-ET treatment. Gynecol Endocrinol. 2013;29:345-9.

20. Grynnerup AGA, Lindhard A, Sørensen S. Anti- Müllerian hormone levels in salpingectomized compared with nonsalpingectomized women with tubal factor infertility and women with unexplained infertility. Acta Obstet Gynecol Scand Suppl. 2013;92:1297-303.

21. Atalay MA, Demir BC, Ozerkan K. Change in the ovarian environment after hysterectomy with bilateral salpingectomy: is it the technique or surgery itself?. Eur J Obstet Gynecol Reprod Biol. 2016;204:57-61. 
22. Song T, Kim MK, Kim ML, Jung YW, Yun BS, Seong SJ et al. Impact of opportunistic salpingectomy on anti- Müllerian hormone in women undergoing laparoscopic hysterectomy: a multicentre randomised controlled trial. BJOG. 2017;124:314-20.

23. Liberati A, Altman DG, Tetzlaff J, Mulrow C, Gøtzsche PC, Ioannidis JP et al. The PRISMA statement for reporting systematic reviews and meta-analyses of studies that evaluate health care interventions: explanation and elaboration. Ann Intern Med. 2009;151:W65-94.

24. Mohamed AA, Al-Hussaini TK, Fathalla MM, El Shamy TT, Abdelaal II, Amer SA. The Impact of excision of Benign Non-Endometriotic Ovarian Cysts on Ovarian Reserve: A systematic review. Am J Obstet Gynecol. 2016;215(2):169-76

25. Raffi F, Metwally M, Amer S. The impact of excision of ovarian endometrioma on ovarian reserve: a systematic review and meta-analysis. J Clin Endocrinol Metab. 2012;97:314654.

26. Iwase A, Nakamura T, Nakahara T, Goto M, Kikkawa F. Assessment of ovarian reserve using anti-Müllerian hormone levels in benign gynecologic conditions and surgical interventions: a systematic narrative review. Rep Biol Endocrinol. 2014;12:125.

27. Fan M, Ma L. Effect of salpingectomy on ovarian response to hyperstimulation during in vitro fertilization: a meta-analysis. Fertil Steril. 2016;106:322-9.e9.

28. Venturella R, Lico D, Borelli M, Imbrogno MG, Cevenini G, Zupi E et al. 3 to 5 Years Later: Long-term Effects of Prophylactic Bilateral Salpingectomy on Ovarian Function. J Minim Invasive Gynecol. 2017;24:145-50.

29. Broekmans FJ, Kwee J, Hendriks DJ, Mol BW, Lambalk CB. A systematic review of tests predicting ovarian reserve and IVF outcome. Hum Reprod Update. 2006;12:685-718.

\section{Supporting Information legends}

Table S1. Studies excluded from the metaanalysis.

Table S2. Search strategies for ovarian reserve after salpingectomy. 


\section{Figure \& Tables Legends}

Table 1. Modified Newcastle Ottawa scale for risk of bias and quality assessment of the included studies.

Table 2. Characteristics of the eight studies included in the meta-analysis.

Table 3 Serum anti-Müllerian hormone (AMH) concentrations after salpingectomy in all eight studies.

Figure 1. PRISMA Flow Chart of the study selection process. AMH, anti-Müllerian hormone.

Figure 2. Weighted mean difference in serum anti-Müllerian hormone concentrations after salpingectomy: pooled results for all six studies in group 1. CI, confidence interval.

Figure 3. Weighted mean difference in serum anti-Müllerian concentrations after salpingectomy: pooled results for two studies in group 2. CI, confidence interval. 
Table 1 Modified Newcastle Ottawa scale for risk of bias and quality assessment of the included studies

\begin{tabular}{|c|c|c|c|c|c|}
\hline Author & Year & $\begin{array}{l}\text { Selection } \\
\text { (***) }\end{array}$ & $\begin{array}{l}\text { Comparability } \\
\qquad(* * *)\end{array}$ & $\begin{array}{l}\text { Outcome } \\
\qquad(* *)\end{array}$ & Overall \\
\hline \multicolumn{6}{|l|}{ Group 1 studies } \\
\hline Findley et al. [9] & 2013 & $* *$ & $* * *$ & $* *$ & 7 \\
\hline Morelli et al. [10] & 2013 & $*$ & $* *$ & $* *$ & 5 \\
\hline Rodgers et al. [11] & 2015 & $*$ & $* * *$ & $* *$ & 6 \\
\hline Venturella et al. [12] & 2015 & $* *$ & $*$ & $* *$ & 5 \\
\hline Sahin et al. [14] & 2016 & $*$ & $* * *$ & $* *$ & 6 \\
\hline Atalay et al. [21] & 2016 & $*$ & $* *$ & $* *$ & 5 \\
\hline \multicolumn{6}{|l|}{ Group 2 studies } \\
\hline Ye et al. [13] & 2014 & $*$ & $* * *$ & - & 4 \\
\hline $\begin{array}{l}\text { Sahin Ersoy et al. } \\
\text { [15] }\end{array}$ & 2016 & $*$ & $* * *$ & $* *$ & 6 \\
\hline
\end{tabular}

This article is protected by copyright. All rights reserved. 
Table 2 Characteristics of the eight studies included in the meta-analysis

\begin{tabular}{|c|c|c|c|c|c|c|c|}
\hline Authors & Year & Country & Design & $\mathrm{n}$ & $\begin{array}{c}\text { Age } \\
\text { mean } \pm S D\end{array}$ & Laterality & Pathology \\
\hline \multicolumn{8}{|l|}{ Group 1 studies } \\
\hline Findley et al [9] & 2013 & USA & Pilot RCT & 15 & $36.6 \pm 4.5$ & bilateral & Normal tubesף[ \\
\hline Morelli et al [10] & 2013 & Italy & $\begin{array}{l}\text { Retrospective } \\
\text { cohort }\end{array}$ & 79 & $45.8 \pm 2.4$ & bilateral & Normal tubes凹 \\
\hline Rodgers et al [11] & 2015 & Australia & Prospective cohort & 20 & $33.06 \pm 4.9$ & unilateral & $\begin{array}{l}\text { Ectopic } \\
\text { pregnancy }\end{array}$ \\
\hline $\begin{array}{l}\text { Venturella et al } \\
{[12]}\end{array}$ & 2015 & Italy & $\mathrm{RCT}$ & 186 & $41.36 \pm 5.39$ & bilateral & Normal tubesश|TI \\
\hline Sahin et al [14] & 2016 & Turkey & Prospective cohort & 61 & $30.9 \pm 4.7$ & unilateral & $\begin{array}{l}\text { Ectopic } \\
\text { pregnancy }\end{array}$ \\
\hline Atalay et al [21] & 2016 & Turkey & Prospective cohort & 103 & $\begin{array}{l}\text { TLH } 42(35- \\
48) \delta \\
\text { TAH } 44(36-48) \\
\delta\end{array}$ & bilateral & Normal tubes \\
\hline
\end{tabular}

\section{Group 2 studies}

Ye et al [13]

2014

China

Retrospective
cohort

$\mathrm{S}=12 \quad 33.2 \pm 4.5$
4

Uni=83

Tubal

$33.8 \pm 4.7$

Bil $=41$

Sahin Ersoy et al [15] 2016 Turkey

Cross-sectional

$$
\mathrm{C}=74
$$

$S=45 \quad 28.3 \pm 3.0$

Unilatera

Ectopic pregnancy

\section{$\mathrm{C}=80 \quad 27.2 \pm 3.5$}

* This study also measured serum AMH level at 4-6 weeks postoperatively

** Tubal pathology (Tuboovarian abscess, ectopic pregnancy, hydrosalpinx)

$\delta$ Data presented as median and range

II Tubes removed during with total laparoscopic hysterectomy

IIII Tubes removed during myomectomy or sterilization

Abbreviations: RCT, randomized controlled trial; $\mathrm{AMH}$, anti-Müllerian hormone; $\mathrm{FSH}$, follicle stimulating hormone; AFC, antral follicle count; OV, ovarian volume; S, salpingectomy group; C, control group; Uni, unilateral; Bil, bilateral; NS, Not specified; $E_{2}$. Estradiol; TLH, total laparoscopic hysterectomy; TAH, total abdominal hysterectomy 
Table 3 Serum AMH concentrations after salpingectomy in all eight studies

\begin{tabular}{|c|c|c|c|c|}
\hline \multicolumn{3}{|l|}{ Author } & \multicolumn{2}{|c|}{$\begin{array}{c}\text { Serum AMH }(\mathrm{ng} / \mathrm{ml}) \\
\text { mean } \pm \text { SD }\end{array}$} \\
\hline Group 1 studies & $\mathbf{n}$ & laterality & Preoperative & Postoperative \\
\hline Findley et al. 2013 [9] & 15 & Bilateral & $2.26 \pm 2.72$ & $1.86 \pm 1.99$ \\
\hline Morelli et al. 2013 [10] & 79 & Bilateral & $0.49 \pm 0.4$ & $0.42 \pm 0.4$ \\
\hline Rodgers et al. 2015 [11] & 20 & Unilateral & $3.52 \pm 2.5$ & $3.59 \pm 3.15$ \\
\hline Venturella et al. 2015 [12] & $\begin{array}{l}\text { Group } A^{*}=91 \\
\text { Group B**=95 } \\
\text { All }=186\end{array}$ & Bilateral & $\begin{array}{l}0.93 \pm 1.13 \\
0.86 \pm 1.01 \\
0.89 \pm 1.07\end{array}$ & $\begin{array}{l}0.83 \pm 1.01 \\
0.79 \pm 0.91 \\
0.80 \pm 0.96\end{array}$ \\
\hline Sahin et al. 2016 [14] & 61 & Unilateral & $2.10 \pm 1.74$ & $2.20 \pm 1.52$ \\
\hline Atalay et al. 2016 [21] & 103 & Bilateral & $1.98 \pm 1.30$ & $1.67 \pm 1.06$ \\
\hline Group 2 studies & $\mathbf{n}$ & Laterality & Study group & Control group \\
\hline Ye et al. 2014 [13] & $\begin{array}{l}S=124 \\
C=74\end{array}$ & $\begin{array}{l}\text { Uni=83 } \\
\text { Bil=41 } \\
\text { Overall=124 }\end{array}$ & $\begin{array}{l}0.02 \pm 0.01 \\
0.01 \pm 0.01 \\
0.01 \pm 0.01\end{array}$ & $0.02 \pm 0.01$ \\
\hline Sahin Ersoy et al. 2016 [15] & $\begin{array}{l}S=45 \\
C=80\end{array}$ & Unilateral & $2.68 \pm 0.59$ & $2.96 \pm 0.85$ \\
\hline
\end{tabular}

*Group A: bilateral salpingectomy without removal of mesosalpinx.

**Group B: bilateral salpingectomy with removal of part of mesosalpinx

Abbreviations: S, salpingectomy group; $\mathbf{C}$, control group; Uni, unilateral; Bil, bilateral 


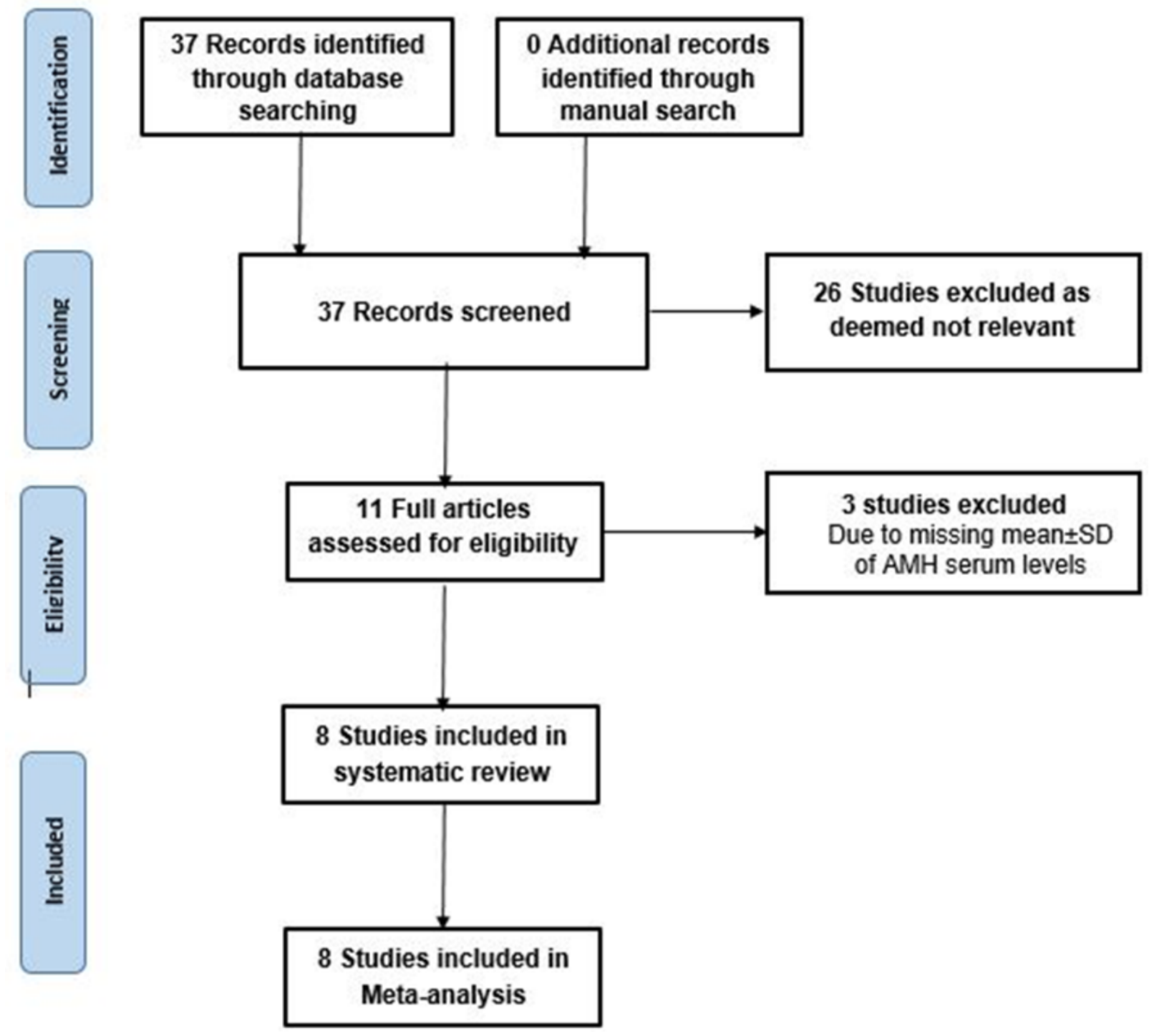

postoperative preoperative Mean Difference Mean Difference Study or Subgroup Mean [ng/ml] SD [ng/ml] Total Mean [ng/ml] SD [ng/ml] Total Weight IV, Fixed, 95\% Cl [ng/ml] Atalay 2016 Findley 2013 Morelli 2013 Rodgers 2015 Sahin 2016

Venturella 2015

$\begin{array}{rrr}1.6 & 1 & 103 \\ 1.86 & 1.99 & 15\end{array}$ $1103 \quad 1.9$

Total $(95 \% \mathrm{Cl})$

Heterogeneity: $\mathrm{Ch}^{2}=255$, df $=5(P=0.77) ; 1^{2}=0 \%$

Test for overall effect: $Z=1.89(P=0.06)$ $\begin{array}{llll}1.9 & 1.2 & 103 & 10.7 \%\end{array}$ $\begin{array}{rrr}1.2 & 103 & 10.7 \% \\ 2.27 & 15 & 0.4 \%\end{array}$ $\begin{array}{rrrr}2.26 & 2.27 & 15 & 0.4 \% \\ 0.49 & 0.4 & 79 & 62.8 \%\end{array}$ $\begin{array}{llll}0.49 & 0.4 & 79 & 62.8 \%\end{array}$ 3.52 $1.74 \quad 61 \quad 2.9 \%$ $\begin{array}{rrr}1.74 & 61 & 2.9 \% \\ 1.07 & 186 & 22.9 \%\end{array}$ $464 \quad 100.0 \%$

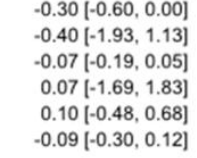

$-0.10[-0.19,0.00]$ 464 0.89

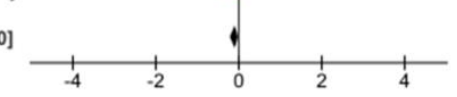


Salpingectomy group

Control group

Mean Difference

Mean Difference

Study or Subgroup Mean $[\mathrm{ng} / \mathrm{ml}] \mathrm{SD}[\mathrm{ng} / \mathrm{ml}]$ Total Mean $[\mathrm{ng} / \mathrm{ml}]$ SD $[\mathrm{ng} / \mathrm{ml}]$ Total Weight IV, Random, $95 \% \mathrm{Cl}[\mathrm{ng} / \mathrm{m}$

Sahin Ersoy 2016

2.

$0.59 \quad 45$

2.96

$\begin{array}{lll}0.85 & 80 & 38.5 \%\end{array}$

$-0.28[-0.53,-0.03]$

IV, Random, $95 \% \mathrm{Cl}[\mathrm{ng} / \mathrm{ml}]$

Total $(95 \% \mathrm{Cl})$

$154100.0 \%$

$-0.01[-0.01,-0.01]$

$-0.11[-0.37,0.14]$

-10

Favours [experimental] Favours [control] 